\title{
Implementation of Extra Efficient Bandwidth Utilization Dynamic Bandwidth Allocation Algorithm to support Differentiated service classes in XGPON
}

Theresal Thangappan

SRM Institute of Science and Technology https://orcid.org/0000-0003-2164-2208

Brintha Therese ( $\square$ abrinthatherese@vit.ac.in )

VIT University - Chennai Campus

\section{Research Article}

Keywords: DBA Algorithm, PON, GPON, XGPON

Posted Date: May 21st, 2021

DOl: https://doi.org/10.21203/rs.3.rs-529888/v1

License: (c) (1) This work is licensed under a Creative Commons Attribution 4.0 International License.

Read Full License 


\section{Abstract}

Dynamic Bandwidth Allocation (DBA) algorithm is necessary for the efficient utilization of bandwidth in XG-PON. Most of the existing DBA algorithms have not utilized the unused bandwidth of a queue with other service classes queue. The Efficient Bandwidth Utilization (EBU) uses a Borrow Refund (BR) method in the update operation to use the unused bandwidth between a traffic class queue of which has some flaws in the BR method. The EBU mechanism causes a reduction in the allocation of bandwidth and increases the delay for the next traffic class. This paper presents an Extra Efficient Bandwidth Utilization (EEBU) algorithm, which overcomes the limitations mentioned above with proper polling and scheduling mechanism. The theoretical and simulation results show that EEBU improvements for T-CONT 2 delay are $1 \%$ and $10 \%$, and for T-CONT 3 delay are $8 \%$ and $22 \%$, and for T-CONT 4 delay by $6 \%$ and $4.5 \%$ compared to the EBU and Giga PON access network (GIANT) respectively.

\section{Introduction}

A telecommunication network which provides the facility of establishing connection between users and their immediate service providers are called Access Networks and it is one of the types of Telecommunication Networks. The optical access network is preferred over other access networks. The service provider uses PON [1] as an access technology that falls in the optical access network category. Optical access network achieves more benefit in terms of distance coverage, speed, cost, etc. PON is a point to multipoint system [2]. PON is found to be much more competent than the Active Optical Network (AON). PON uses passive splitter and combiner and optical fiber. PON further classified as Asynchronous Transfer Mode (ATM) PON (APON), Broadband PON (BPON), Ethernet PON (EPON), GPON, and Wavelength Division Multiplexing PON (WDM-PON) [3].

GPON is preferred among all the other passive optical networks because of its data rate, split ratio, Quality of Service (QoS), and wide applications. Features like high efficiency, rich user interface, high bandwidth, and wide coverage make it a perfect technology to realize broadband access network services. It operates in the downstream and upstream direction at a wavelength of 1480-1500 nm and $1260-1360 \mathrm{~nm}$. With a scalable bandwidth from $155 \mathrm{Mbit} / \mathrm{s}$ to $2.5 \mathrm{Gbit} / \mathrm{s}$. It provides a maximum downstream speed of $2.488 \mathrm{~Gb} / \mathrm{s}$ and an upstream speed of $1.244 \mathrm{~Gb} / \mathrm{s}$. The physical network support reaches up to $60 \mathrm{~km}$ and supports a split ratio of up to 128 based on standards. GPON has Asynchronous Transfer Mode (ATM) or GPON encapsulation method (GEM) to transport data. But GEM is the most preferred one. It works on the IP protocol. Transmission Containers (T-CONTs) handle the upstream bandwidth allocation. Each T-CONT type will have a separate queue, and each queue will have a different Alloc-ID (Allocation Identifier) and will be maintained overall by ONUs. T-CONT 1, T-CONT 2, T-CONT 3, TCONT 4, T-CONT 5 are the five T-CONTs [6]. In Table 1 shows the T-CONT types, parameters, and application. 
Table 1

T-Cont types and parameters

\begin{tabular}{|llll|}
\hline T-CONT types & Bandwidth & Parameters & Application \\
\hline T-CONT 1 & Fixed & $\mathrm{SI}_{\text {max }}, \mathrm{AB}_{\text {min }}$ & Leased lines \\
\hline T-CONT 2 & Assured & $\mathrm{SI}_{\text {max }}, \mathrm{AB}_{\text {min }}$ & Voice \\
\hline T-CONT 3 & Assured & $\mathrm{SI}_{\text {max }}, \mathrm{AB}_{\text {min }}$ & Video \\
\cline { 2 - 3 } & Non-assured & $\mathrm{SI}_{\text {min }}, \mathrm{AB}_{\text {sur }}$ & \\
\hline T-CONT 4 & Best effort & $\mathrm{SI}_{\text {min }}, \mathrm{AB}_{\text {sur }}$ & Email, Browsing \\
\hline T-CONT 5 & Mixed & No parameters & No application \\
\hline
\end{tabular}

GPON is subdivided into XGPON and Next Generation PON (NGPON). The difference between these and GPON is based on data rates. In XGPON, the main advantage is that the bitrates increased downstream to $10 \mathrm{~Gb} / \mathrm{s}$ and upstream $2.5 \mathrm{~Gb} / \mathrm{s}$ compared with GPON [7]. GPON and XGPON follow the DBA approach. Every algorithm has an advantage. DBA algorithm has a function of allocating and sharing the excess bandwidth collected from the lightly loaded to the heavily loaded unit [8]. GPON physical reach is about $60 \mathrm{~km}$ and achieves a split ratio of up to 256 , and is almost double the number of GPON [9]. Apart from these differences, there are many similarities between GPON and XGPON standards like its upstream bandwidth DBA scheme, medium sharing TDMA mechanism, QOS service methodology, etc. Based on the observation, it seems that XGPON could help the evolution of many intensive-bandwidth applications like IPTV, Video conference, chatting, or video messages. In cellular networks, XG-PON is used as the backhaul and front haul interface for MBS (Multiple Base Stations) and provides faster network facilities in mobiles by integrating with various wireless networks [10].

The rest of the paper ordered as follows. Section II reviews the related work, section III explains the polling and scheduling mechanism of the proposed EEBU algorithm, and section IV describes the result and comparison with the existing few algorithms. Lately. Section $V$ concludes the future works related to further research.

\section{li. Related Papers}

The First DBA algorithm for GPON was introduced in 2006[11]. The working: It has two sections, the Guaranteed Phase Allocation (GPA) and the Surplus Phase Allocation (SPA). The SPA begins after the execution of the GPA. GPA is executed for T-CONT2 and T-CONT3 since T-CONT2 and T-CONT3 require assured bandwidth. T-CONT3 requires non- guaranteed bandwidth as well. Parameters such as Service Interval ( $\mathrm{SI}$ ) and Available Bandwidth (AB) are necessary for each T-CONT to assign bandwidth and vary for each T-CONT. 
GIANT uses the Down counter and its involvement in allocating the bandwidth to each of the service classes. Its value is diminished by unity for every frame duration (FD), and on expiration, the bandwidth is allocated. GIANT algorithms have an idle time delay, and at last, there is a bandwidth that remains unallocated. To overcome the GIANT algorithm's idle time delay, the Immediate Allocation with Colorless Grant (IACG) algorithm [12] was introduced. The available byte counter (VB) is used in each ONU for each service class, and allocated bytes are stored for that class. The unused bandwidth is assigned to each of the ONU by using T-CONT5. Still, the unused bandwidth exists. For this, the EBU algorithm [13] is introduced, where unutilized bandwidth is used by the borrow and refund (BR) method in the update operation. The available byte counter changes to negative if the demand is greater than its VB size, then it borrows the bandwidth from the available byte counter of other traffic. Refunds it from the common counter in update operation, suppose if there is no bandwidth available in common counter, it leads to reduced bandwidth allocation to the remaining traffic classes. To address the shortcomings of the EBU algorithm, the Simple and Feasible DBA algorithm (SFDBA) [14] was developed. It has a single available byte counter for all traffic classes. However, if that service class's byte counter gets depleted, a different service class cannot use one service class's unused bandwidth. To address these issues, the DBA with High Utilization (DBAHU) algorithm [15] was introduced as an updated version of SFDBA. In the SFDBA update operation, the DBAHU algorithm employs the BR concept of EBU.

Furthermore, in the case of no unused bandwidth, the next cycle is affected. The X-GIANT algorithm [16] improves on the GIANT concept in the XGPON standard by allocating bandwidth regardless of the expiry of the down counter. The enhanced Bandwidth Utilization (IBU) algorithm [17] enhances the polling and scheduling mechanisms. IBU increases the T-CONT 4 delay by giving the most priority to T-CONT2 and TCONT3. The Comprehensive Bandwidth Utilization (CBU) algorithm [18] is useful in improving the IACG and EBU polling and scheduling mechanisms. It improves the T-CONT4 traffic delay but fails in T-CONT2 and T-CONT3 traffic compared to EBU. A new polling and scheduling mechanism used for the CBA algorithm [19]. Demand Forecasting (DF) DBA [20] is based on statistical modelling, and the OLT predicts ONU traffic demands. The DF-DBA algorithm reduces the time required to process the ONU request and DBA calculation. Thus the time for processing the ONU request and DBA calculation is eliminated. In the literature review, it is clear that XGPON 's current DBA algorithms do not effectively and efficiently allocate the remaining bandwidth in the available byte counter. This paper introduced a new algorithm named EEBU to utilize unused bandwidth completely without any complexity.

\section{lii. Eebu Algorithm}

\section{A. EEBU Polling Mechanism:}

The polling and scheduling mechanism follows Service SI. RTT/2 is the time taken by the OLT to assign allocation to $\mathrm{ONU}$ and vice versa. Once the Dynamic Bandwidth Request upstream $\left(\mathrm{DBR}_{\mathrm{u}}\right)$ slot reaches the $\mathrm{ONU}$, it takes $\mathrm{T}_{0}$ time plus $\mathrm{T}_{\mathrm{E}}$ time for processing the request from queues, where $T_{0}$ is the processing delay, and $T_{E}$ is the equalization delay. The request processing from ONUs is called polling. The request processed from ONUs is sent to OLT. After reaching OLT, it takes $\mathrm{T}_{\mathrm{OLT}}$ time to calculate the demand and 
provide it according to the request. $\mathrm{T}_{\mathrm{OLT}}$ consists of $\mathrm{T}_{\mathrm{DBA}}$ time to process the DBA algorithm and GRANT time to allocate grants. Allocating grants to ONUs is called scheduling. Usually, SI is multiples of $125 \mu \mathrm{s}$. $\mathrm{SI}$ is divided into types of $\mathrm{SI}_{\mathrm{MAX}}$ and $\mathrm{SI}_{\mathrm{MIN}}$ depending upon the service class. The Allocation Bytes depends upon the service rate $(\mathrm{R})$ and $\mathrm{SI}$.

T-CONT of each ONU are T-CONT2, T-CONT3, and T-CONT4, T-CONT has one queue with an Alloc-ID. The number of cycles depends upon the value of SI. The previous cycle grant is allocated with the $\mathrm{DBR}_{\mathrm{u}}$ slot in $\mathrm{C} 0$ and reaches the $\mathrm{ONU}$ at $\mathrm{C} 1$. The request report collected from the ONUs during $\mathrm{C} 1$ sent to the OLT during $\mathrm{C} 2$. At $\mathrm{C} 3$, all the reports go to the OLT. The OLT processes the request and assigns the grant during $\mathrm{C} 4$. It reaches the $\mathrm{ONU}$ at $\mathrm{C} 1$ of the next cycle or $\mathrm{C} 5$ of the current cycle. But when the report reaches $\mathrm{C} 2$, it is used in $\mathrm{C} 3$. By this time, the $\mathrm{ONU}$ has consumed the allocations $\mathrm{G} 0, \mathrm{G} 1, \mathrm{G} 2$, and $\mathrm{G} 3$ from four cycles $\mathrm{C} 0, \mathrm{C} 1, \mathrm{C} 2$, and $\mathrm{C} 3$. These allocations subtracted from the report before processing them for a grant. Figure 1 shows the XGPON transmission diagram during the polling process.

Each queue is assigned with a Polling flag by the OLT. Polling flag $P F_{K J}$ is open i.e., set to 0 , the OLT allocates a $\mathrm{DBR}_{\mathrm{u}}$ slot to that queue during downstream. The variable $\mathrm{DBR}_{\mathrm{KJ}}$ denotes the $\mathrm{DBR}_{\mathrm{u}}$ slot assigned to the queue $Q_{K J}$. The queue can place it's requested in that $D B R_{U}$ slot and sends the slot upstream. Once a $D B R_{u}$ slot is allocated, the $D_{B R}$ flag is closed i.e., set to 1. The $D_{B R}$ flag is opened again when the SI timer expires. The proposed algorithm gives one $\mathrm{DBR}_{\mathrm{U}}$ slot per SI. To know the current request of queues, it must report every downstream cycle, but allocating $D B B R_{u}$ slot for every Upstream cycle leads to bandwidth wastage as the $\mathrm{DBR}_{\mathrm{u}}$ slot length is 4 bytes for one queue. The polling flag is opened again when the SI timer expires. The proposed algorithm allocates one $\mathrm{DBR}_{\mathrm{u}}$ slot per SI. Each ONU has three queues, so $12 * \mathrm{~W}$ bytes are wasted where $\mathrm{W}$ represents the ONU number. 


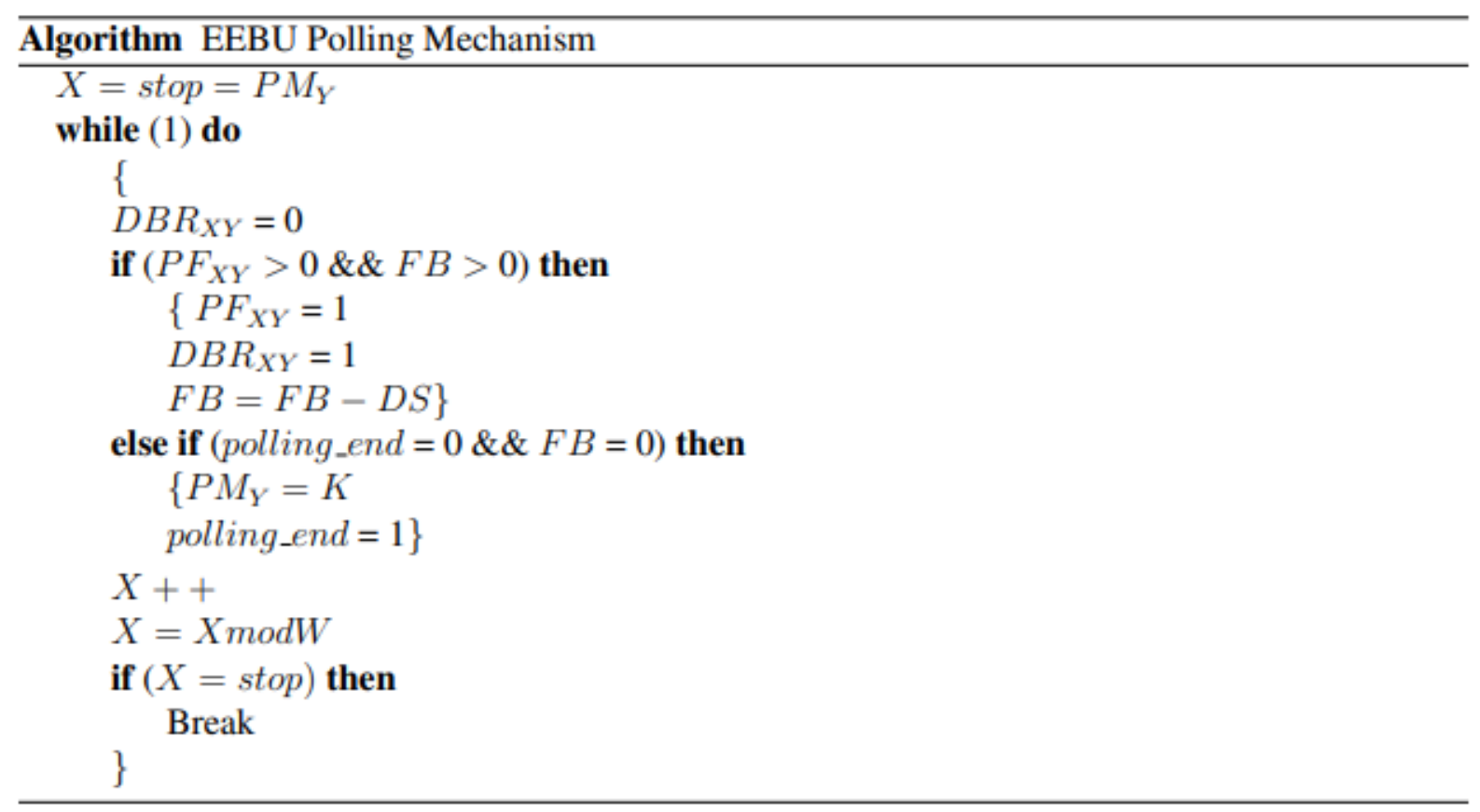

$\mathrm{PM}_{\mathrm{Y}}$ indicates the ONU in which polling begins for T-CONT type $\mathrm{Y}$. The polling mechanism is operated according to SLA. DBR $\mathrm{XY}_{\mathrm{Y}}$ denotes the $\mathrm{DBR}_{\mathrm{u}}$ slot for T-CONT type $\mathrm{Y}$ of ONU X. DBR $\mathrm{R}_{\mathrm{XY}}=0$ means the slot is not assigned. The variable $\mathrm{PF}_{\mathrm{XY}}$ represents the $\mathrm{DBR}_{\mathrm{u}}$ flag for T-CONT type $\mathrm{Y}$ of $\mathrm{ONU} \mathrm{X}$. $\mathrm{PF}_{\mathrm{XY}}=0$ means the polling flag is open. $D B R_{X Y}=1$, means the slot is assigned. $D S$ denotes the size of the $D_{B R}$ slot. polling_end indicates the termination of a polling mechanism. If polling_end $=1$ means polling is ended.

\section{B. EEBU Scheduling mechanism}

The scheduling mechanism in the proposed DBA algorithm follows SLA. The request report was obtained from the polling operation used in scheduling for allocating bandwidth. EEBU algorithm uses a common counter $(T)$, which stores the values of the available byte counter of all T-CONT types $\left(V B_{Y}\right)$. All the traffic queue uses bandwidth from the common counter, and the unutilized bandwidth of a queue will be shared with other queues. In this way, it eliminates the borrow refund operation in the update operations-the granting starts when $T$ and Frame Bytes(FB) are greater than zero. Then the minimum condition, the OLT, grants the minimum of Request of T-CONT type $\mathrm{Y}$ of ONU $\mathrm{X}\left(\mathrm{R}_{\mathrm{XY}}\right), \mathrm{T}$, and FB. Then the grant size is subtracted from these factors. Finally, the update operation starts when the Down counter of traffic class $Y\left(D_{Y}\right)$ expires. This $V B_{Y}$ is recharged to Allocated Bandwidth for T-CONT type $Y\left(A B_{Y}\right), D_{Y}$ recharged to $S I_{Y}$, and the $P F_{X Y}$ opened. If allocation_end $=1$ means allocation is ended. For XGPON, the value for frame bytes is 38880 bytes. $A B_{X Y}$ denotes the allocation bytes for T-CONT type $Y$ of ONU X. SI $I_{X Y}$ denotes the service interval for T-CONT type $\mathrm{Y}$ of ONU X. 


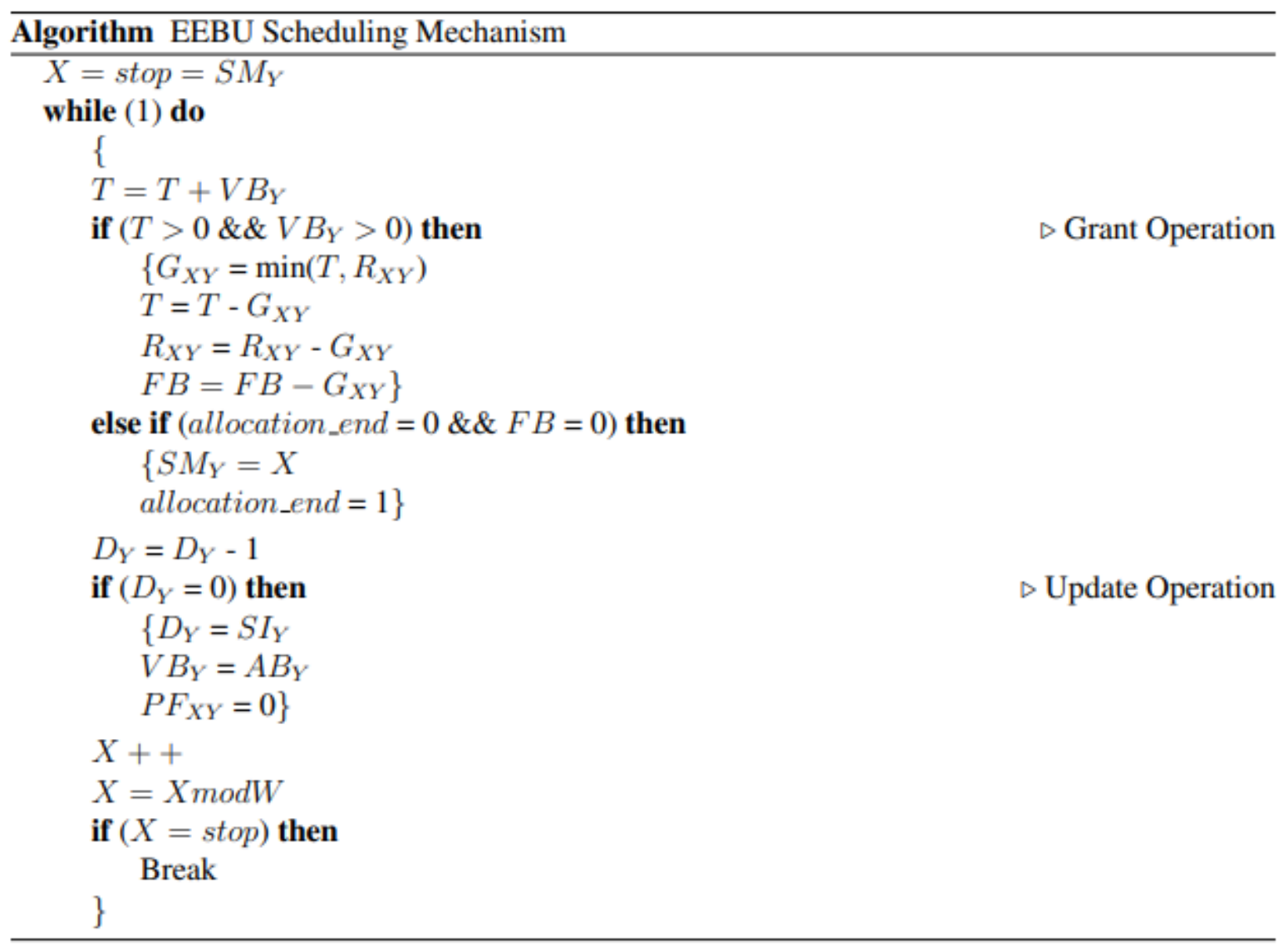

\section{Theoretical Analysis}

The theoretical analysis made to show EEBU outperforms EBU in utilizing unused bandwidth completely. Consider there are two ONU's in the system. Let request R12 and R22 be the request of ONU1 and ONU2 of T-CONT type 2, considering there is no request from ONU1 and ONU 2 for T-CONT type 2. Let R13 and R23 request ONU1 and ONU2 of T-CONT type 3 and assume it is 50 and 100 bytes. Let V2 and V3 be the byte counter for T-CONT types 2 and T-CONT type 3 , and consider it is 200 and 50 bytes. The process starts from ONU 1 and then ONU 2 of T-CONT type 2, followed by ONU1 and ONU 2 of T-CONT type 3. Since the request R12 and R22 are zero, no grant is issued. The R13 granted, and VB3 becomes zero. But R23 cannot be given because there are no bytes in VB3, and also because of its limitation, it cannot use VB2. The Bytes in VB2 are unused, and it gets wasted. In EEBU, the bytes allocated in VB2 have added to T. The grant is issued only by $T$. Since the request R12, R22 is zero, no grant given. Now the bytes of VB3 are added to $T(200+50)$. R13 granted, and T becomes 200 . Next, R23 granted, $T$ becomes 100 . By this example, we consider that the unused bandwidth is efficiently utilized in the EEBU algorithm.

\section{Simulation Model and Conditions}

The simulation tool used in this paper is NS-3. NS-3 is a network simulator [21] that uses C ++ and Python. The XG-PON module was developed with NS-3[22]. In this paper, the simulation scenario consists 
of $16 \mathrm{ONUs}(\mathrm{W})$ and 1 OLT. Table 2 displays the parameters taken for simulation and their values for the algorithm. Each ONU contains 3 T-CONTs (2, 3, and 4). It offers traffic loads from 0.1 to 1 . The queue size for each T-CONT is $1 \mathrm{MB}$ (Megabytes). The rates on the upstream and downstream are set at $2.488 \mathrm{Gbps}$ and $10 \mathrm{Gbps}$. The Optical Distribution Network area is $20 \mathrm{Km}$, so RTT is $200 \mu \mathrm{s}$. The processing time of $\mathrm{ONU}(\mathrm{TO})$ is $35 \mu \mathrm{s}$.

Table:2 Simulation parameter and values

\begin{tabular}{|ll|}
\hline Parameter & Value \\
\hline OLT & 1 \\
\hline No of ONUs & 16 \\
\hline Traffic Load offered & 0.1 to 1 \\
\hline Upstream data rate & $2.5 \mathrm{Gbps}$ \\
\hline Downstream data rate & $10 \mathrm{Gbps}$ \\
\hline Size of the Packet & 1472 bytes \\
\hline TCONT-type & 2,3, and 4 \\
\hline Environment Area & $20 \mathrm{Km}$ \\
\hline
\end{tabular}

\section{Iv. Result And Discussion}

Delay is a time taken by the packet to reach the destination from the source. The source indicates the OLT and destination indicates the ONU. The results measured a delay in milliseconds. Figure 2 shows the average delay values of 16 ONUs under various network loads for Traffic classes 2, 3, and 4 and total upstream.

Figure 2(a), for T-CONT 2, the delay performance of EEBU is similar to EBU, since ONU's bandwidth allocation process starts from the T-CONT 2 traffic class. However, at the middle load, when the traffic load is equal to 0.5 , EEBU's delay performance is increased by 1 percent due to the total counter's use. Compared to the GIANT algorithm, at load offered is equal to 0.3 , the delay performance increased around $10 \%$. When the traffic load is equal to 0.5 , the delay performance improved by $7.5 \%$, and the remaining traffic load maintained the delay performance around $4 \%$. The maximum and minimum T2 delay in EEBU is reduced by $10 \%$ and $4 \%$ compared to the GIANT.

More priority goes to T-CONT 3 since it is of video application. Figure 2(b) shows that the T-CONT 3 delay performance of the EEBU is improved by $8 \%$ at the traffic load equal to 0.3 , compared to EBU. It maintains the constant delay improvement for the remaining traffic load of around $4 \%$. Compared to GIANT, the initial traffic load at 0.1 to 0.3 nearly $22 \%$ delay improvement and maintains a $7 \%$ improvement for the remaining traffic load. Compared to EBU, the maximum and minimum T-CONT 3 delay in EEBU is reduced 
by $8 \%$ and $4 \%$ by eliminating borrow refund operation in T-CONT 2 traffic class. Compare to GIANT, the maximum and minimum T-CONT 3 delay in EEBU reduced by $22 \%$ and $7 \%$.

From Fig. 2(c), the delay performance of EEBU is improved over EBU for T-CONT 4 traffic class at middle and higher loads because of the removal of borrow refund operation in the update operation. The T-CONT 4 delay in EEBU is reduced by $5.9 \%$ compared to EBU. In GIANT delay performance is similar to EEBU, the middle, the delay performance improved by $4.5 \%$.

Figure 2(d) shows that the upstream delay is the average value of the sum of T-CONT 2, T-CONT 3, and TCONT 4 class delay. The upstream delay of EEBU improved over EBU as the delay of T-CONT 2, T-CONT 3, and T-CONT 4 traffic class in EEBU is less than EBU and GIANT. The maximum and minimum delay in EEBU is reduced by $2 \%$ and $2.6 \%$ compared to EBU, and $11 \%$ and $3 \%$ compared to GIANT.

Hence, the simulation results prove that EEBU reduces the delay of T-CONT 2 traffic class by $1 \%$, T-CONT 3 traffic class by $4 \%$, for T-CONT 4 traffic class by $5.9 \%$, and upstream delay is by $2 \%$ compared to EBU. Compared to the GIANT, the delay performance of T-CONT 2 traffic class by $4 \%$, T-CONT 3 traffic class by $7 \%$, for T-CONT 4 traffic class by $4.5 \%$, and upstream delay is reduced by $2.6 \%$.

\section{Conclusion}

In this paper, the fiber access network is preferred over other access networks. PON is chosen over other technology in optical Fiber because of its shared architecture. Due to better features, GPON is preferred over EPON. XG-PON is adopted for this study as it's the updated version of GPON, and its details presented in this report. A study performed in XGPON on the DBA algorithms showed that the available bandwidth was not used adequately. This paper proposed a new model of the DBA algorithm for XG-PON. It improves the EBU algorithm's scheduling mechanism, where the unused bandwidth is adequately utilized by the total counter so that there is no leftover. The delay of individual traffic classes and overall upstream delay of EEBU is reduced compared to EBU and GIANT. Therefore, the EEBU algorithm can support triple-play services more efficiently compared to the existing DBA algorithms from the results and analysis. This project will be useful for evaluating certain futuristic technologies like front haul and backhaul in $5 \mathrm{G}$.

\section{Declarations}

We hereby declare that we are the sole author's of this article. To the best of my knowledge this article contains no material previously published by any other person except where due acknowledgement has been made.

\section{References}

1. Effenberger, F., Cleary, D., Haran, O., Kramer, G., Li, R. D., Oron, M., \& Pfeiffer, T. An introduction to PON technologies [Topics in Optical Communications]. IEEE Communications Magazine. 2007 Apr 
10;45(3): S17-25.

2. Lee, C. H., Sorin, W. V., \& Kim, B. Y. Fiber to the home using a PON infrastructure. Journal of lightwave technology. 2006 Dec 1;24(12):4568-83.

3. Vukovic, A., Savoie, M., Hua, H., \& Maamoun, K. Performance characterization of PON technologies. In Photonics North 20072007 Oct 30 (Vol. 6796, p. 67962W). International Society for Optics and Photonics.

4. Abbas, H. S., \& Gregory, M. A. (2016 May). The next generation of passive optical networks: A review. Journal of Network and Computer Applications, 67(1), 53-74.

5. ITU-T. Gigabit-capable passive optical networks (GPON): General characteristics, 2018.

6. 6.Recommendation IT. 987.3, 10-Gigabit-capable passive optical networks (XG-PON): Transmission convergence (TC) layer specification. Oct-2010.

7. ITU-T, 10-Gigabit-Capable Passive Optical Networks (XG-PON): General Requirements, ITU-T Recommendation G.987.1. E 40850, (2010).

8. TheresalThangappan, BrinthaTherese,SuvarnammaAdi, Sai SwapnaGadda. Review on Dynamic Bandwidth. (2020). Allocation of GPON and EPON. Journal of Electronic Science andTechnology.

9. ITU-T, 10-Gigabit-capable passive optical networks (XG-PON): Physical media dependent (PMD) layer specification G.987.2. E 40850, (2012).

10. ITU-T, -5G wireless front haul requirements in a passive optical network context SERIES G, supplement 66, (2019), pp. 1-40.

11. Leligou, H. C., Linardakis, C., Kanonakis, K., \& Angelopoulos, J. D. (2006). and T.Orphanoudakis, Efficient medium arbitration of FSAN compliant GPONs. International Journal of Communication Systems, 19(5), 603-617.

12. Han, M. S., Yoo, H., Yoon, B. Y., Kim, B., \& Koh, J. S. (2008). Efficient dynamic bandwidth allocation for FSAN-compliant GPON. Journal of Optical Networking, 7(8), 783-795.

13. Han, M. S., Yoo, H., \& Lee, D. S. Development of efficient dynamic bandwidth allocation algorithm for XGPON, ETRI Journal, vol. 35, no. 1, pp. 18-26, 2013.

14. Han, M. S., Simple and Feasible Dynamic Bandwidth and Polling Allocation for XGPON, in ICACT Transactions on Advanced Communications Technology (TACT), vol. 2, no. 5, pp. 298-304, 2013.

15. Han, M. S., Dynamic bandwidth allocation with high utilization for XGPON, in: 16th International Conference in Advanced Communication Technology, IEEE; 2014: 994- 997.doi: https://doi.org/10.1109/ICACT.2014.6779107.

16. Arokkiam, J. A., \& Brown, K. N., Refining the GIANT dynamic bandwidth allocation mechanism for XGPON, in IEEE ICC 2015 SAC - Access Networks and Systems Refining, pp. 2609-2614, 2015.

17. Butt, R. A., \& Idrus, S. M. (2017). K. N. Qureshi. Improved dynamic bandwidth allocation algorithm for XGPON. Journal of Optical Communication Networking, 9(1), 87-97. https://doi.org/10.1364/JOCN.9.000087. 
18. Butt, R. A., Idrus, S. M., Rehman, S. U., Shah, P. M. A., \& Zulkifli, N. Comprehensive polling and scheduling mechanism for long reach gigabit passive optical network. Journal of Optical Communication.2017;0(0):1-12. https://doi.org/10.1515/joc-2017-0026.

19. Butt, R. A., Idrus, S. M., Zulkifli, N., \& Waqar, M., \& Ashraf (2017). Comprehensive bandwidth utilization and polling mechanism for XGPON. International Journal of Communication Systems, no., 31(3), 118.

20. Memon, A. K., Mohammadani, H. K., Ain, U. N., Shaikh, A., Ullah, S., Zhang, Q., Das, B., Ullah, R., Tian, F., \& Xin, X. Demand forecasting DBA algorithm for reducing packet delay with efficient bandwidth allocation in XG-PON, Electronics 8 (2019).

21. Riley, G. F., \& Henderson, T. R. (2010). The ns-3 Network Simulator. In K. Wehrle, M. Güneş \& J. Gross (Eds.), Modeling and Tools for Network Simulation. Berlin: Springer.

22. Wu, X., Brown, K. N., Sreenan, C. J., Alvarez, P., Ruffini, M., Marchetti, N., Payne, D., \& Doyle, L. 2013. An XG-PON module for the NS-3 network simulator.

\section{Figures}

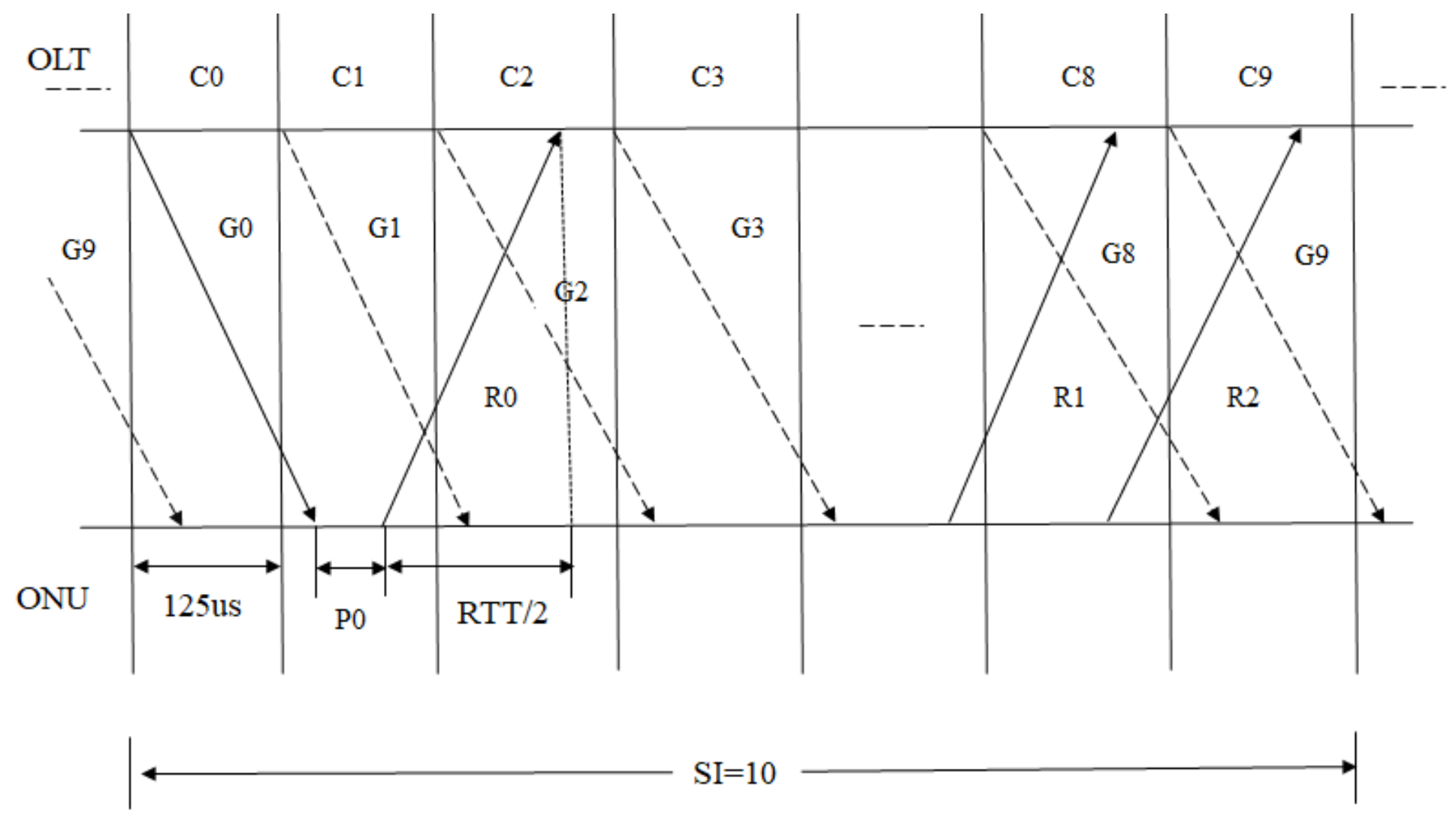

Figure 1

XGPON Transmission Diagram 


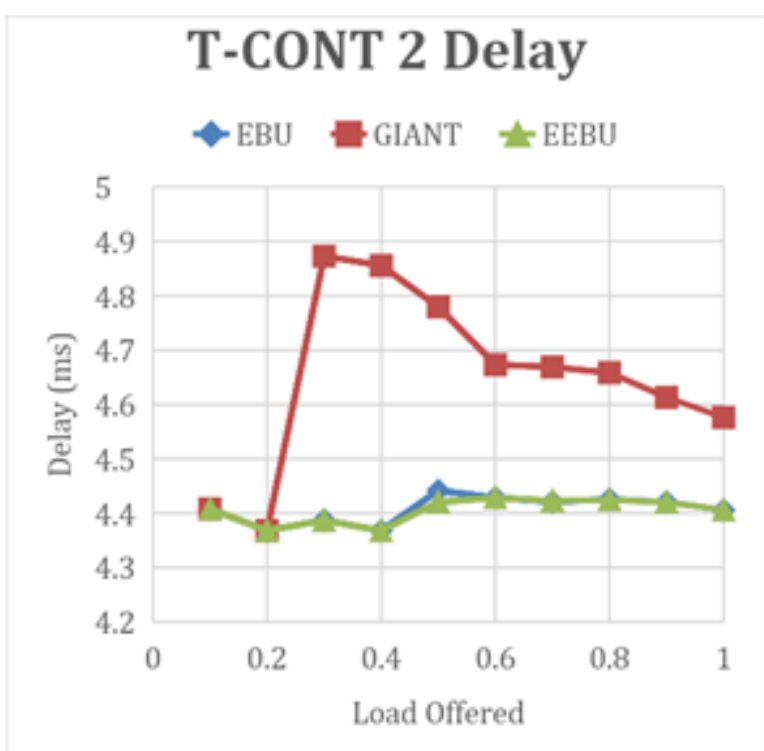

(a)

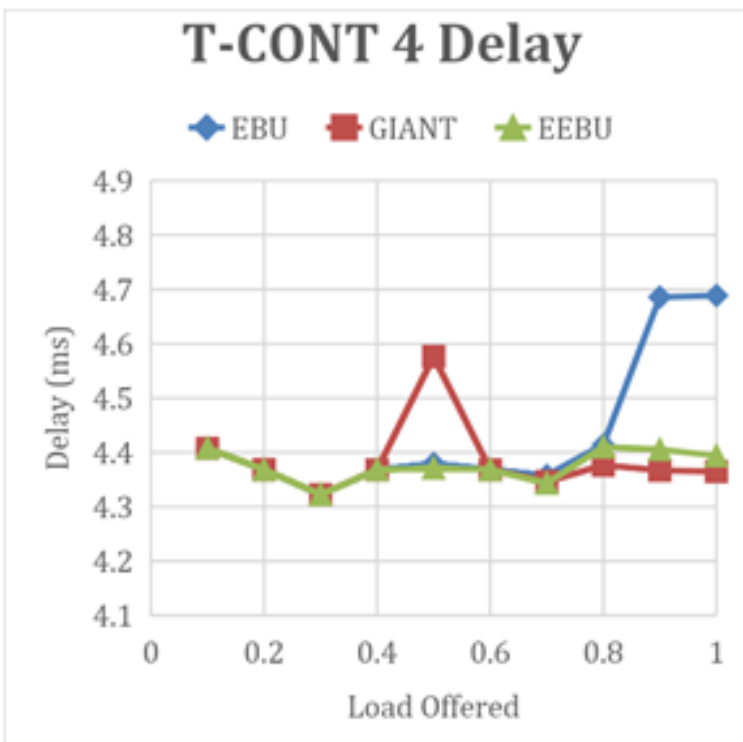

(c)

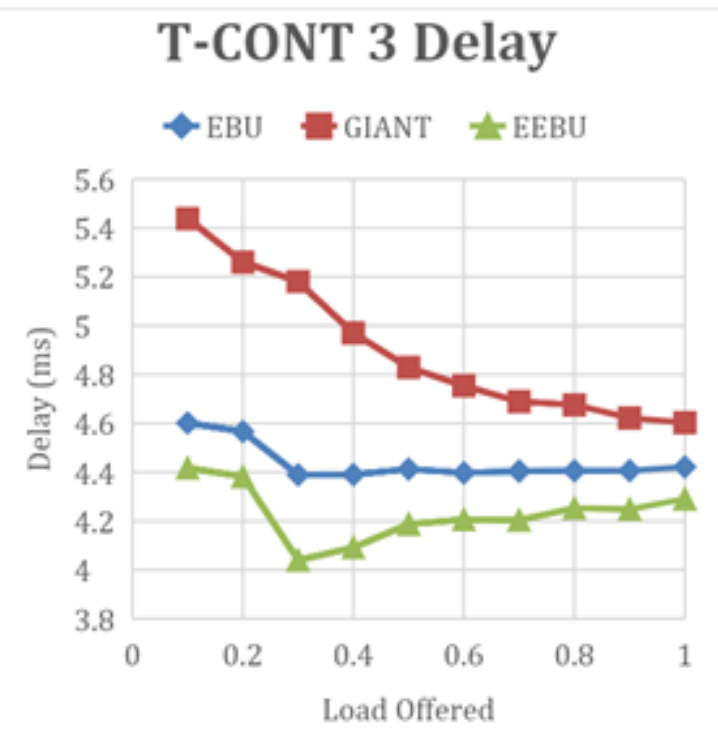

(b)

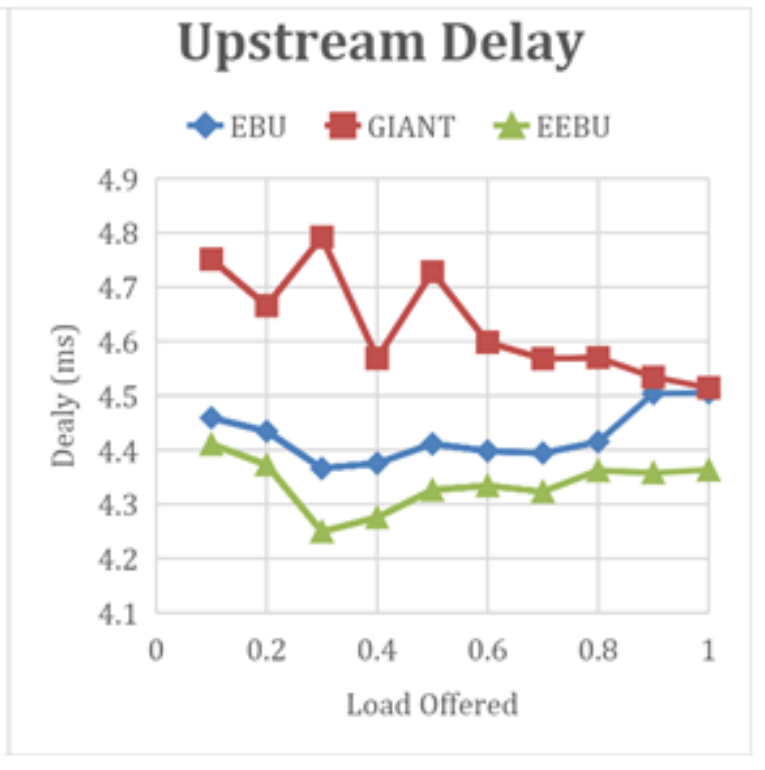

(d)

Figure 2

Traffic delay for different classes (a) T-CONT 2, (b) T-CONT 3, (c)T-CONT 4, and (d) Upstream delay 